\title{
Anharmonic effect on the equation of state (EoS) for $\mathrm{NaCl}$
}

\author{
Tatsuya Sumita $\cdot$ Akira Yoneda
}

Received: 11 April 2013 / Accepted: 16 September 2013 / Published online: 16 October 2013

(C) The Author(s) 2013. This article is published with open access at Springerlink.com

\begin{abstract}
We find clear intrinsic anharmonicity in the $\mathrm{NaCl}-\mathrm{B} 1$ phase by examining the equation of state (EoS) based on previous ultrasonic velocity data for pressures up to $0.8 \mathrm{GPa}$ and temperatures up to $800 \mathrm{~K}$. The experimental EoS for this phase shows that its specific heat at constant volume $\left(C_{V}\right)$ is significantly smaller than that based on a harmonic model. Also, the sign of $\left(\partial C_{V} / \partial P\right)_{T}$, which is normally negative in the quasi-harmonic approximation, is unexpectedly positive. The thermodynamic Grüneisen parameter $(\gamma)$, which has frequently been assumed to be a single-variable function of molar volume, shows not only volume dependence but also negative temperature dependence. To understand these features of $C_{V}$ and $\gamma$, we introduce a thermodynamic model including positive quartic anharmonicity. To make an anharmonic model advancing the ordinarily quasi-harmonic approximation model, we introduce two parameters: anharmonic characteristic temperature $\left(\theta_{a}\right)$ and its volume derivative. In the anharmonic model, the value of $C_{V}$ is calculated along an isochore using classical statistical mechanics and a harmonic quantum correction. At high temperatures, the decrease in $C_{V}$ from the Dulong-Petit limit is related to the value of $T / \theta_{a}$. For infinitely large $\theta_{a}$, the system is
\end{abstract}

Electronic supplementary material The online version of this article (doi:10.1007/s00269-013-0627-z) contains supplementary material, which is available to authorized users.

T. Sumita $(\bowtie)$

Geological Survey of Japan, National Institute of Advanced Industrial Science and Technology (AIST), Tsukuba, Ibaraki 305-8567, Japan

e-mail: t.sumita@aist.go.jp

A. Yoneda

Institute for Study of the Earth's Interior, Okayama University, Misasa, Tottori 682-0193, Japan approximately quasi-harmonic. The temperature dependence of $\gamma$ is related to $C_{V}$ by the thermodynamic identity $\left(\partial C_{V} / \partial \ln V\right)_{T}=C_{V}(\partial \gamma / \partial \ln T)_{V}+\gamma\left(\partial C_{V} / \partial \ln T\right)_{V}$. Even though our modification of the quasi-harmonic approximation is simple, our anharmonic model succeeds in reproducing the experimental $\gamma$ and $C_{V}$ simultaneously for the NaCl-B1 phase.

Keywords Complete travel-time equation of state (CT-EoS) - Intrinsic anharmonicity · Thermodynamic model $\cdot$ Statistical mechanics $\cdot \mathrm{NaCl}-\mathrm{B} 1$ phase $\cdot$ Grüneisen parameter

\section{Introduction}

The equation of state (EoS) for the NaCl-B1 phase is representative of pressure scales up to approximately $25 \mathrm{GPa}$. In particular, Decker's (1971) EoS is based on both lattice dynamics and the quasi-harmonic Debye model and has frequently been used for more than 30 years in high-pressure experiments in solid-state physics, geophysics, and material sciences. However, Decker's (1971) EoS cannot reproduce the corresponding zero-pressure thermodynamic properties (e.g., thermal expansion coefficient $\alpha(0, T)$ and isobaric specific heat $C_{P}(0, T)$; compare the thin solid line with the connected circles in Fig. 1). Later, Brown (1999) reported an EoS that was consistent with experimental $\alpha(0$, $T)$ data. However, the $C_{P}(0, T)$ values are still significantly inconsistent with experiment (see dashed line in Fig. 1). These two EoSs commonly assume both the quasi-harmonic Debye model and a $\gamma$ value that is dependent solely on molar volume, i.e., $\gamma=\gamma(V)$.

Spetzler et al. (1972) found experimental support for the $\gamma=\gamma(V)$ hypothesis. They measured the ultrasonic velocity 


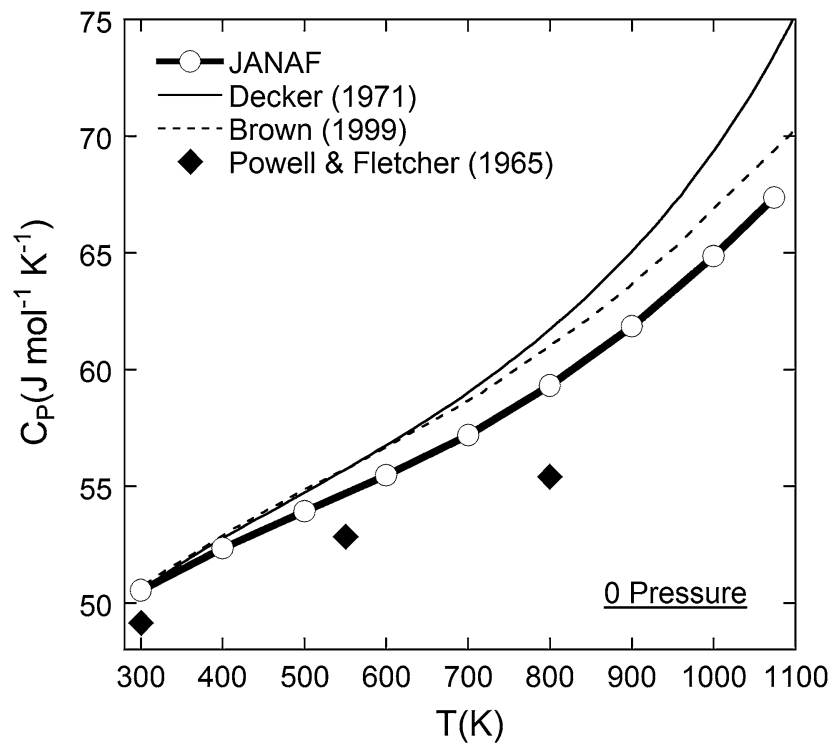

Fig. 1 Comparison of the isobaric specific heat at zero pressure. Bold line with circles experimental values compiled by Chase (1998); thin solid line Decker (1971) EoS; dashed line Brown (1999) EoS; diamonds theoretical values of Powell and Fletcher (1965) as used in Spetzler et al. (1972)

at zero pressure and $300 \mathrm{~K}, v_{i}(0,300 \mathrm{~K})$, and the frequency (normalized inverse travel time) up to $0.8 \mathrm{GPa}$ and $800 \mathrm{~K}$, $F_{i}(P, T)$, and then calculated major thermodynamic quantities including $\gamma$ from the ultrasonic data combined with $\alpha(0, T)$ and $C_{P}(0, T)$. Spetzler and Yoneda (1993) refer to this calculated result as the complete travel-time equation of state, or CT-EoS. However, we have ascertained that Spetzler et al. (1972) calculated the CT-EoS using $C_{P}(0, T)$ values based on a theoretical model (Powell and Fletcher $1965)$ that yields values $7 \%$ smaller than the experimental (Chase 1998) at $800 \mathrm{~K}$ (see Fig. 1). These unsatisfactory $C_{P}$ values cause significant uncertainty in $\gamma$, because $\gamma=\alpha V K_{S} / C_{P}$ (where $K_{S}$ is the adiabatic bulk modulus).

Therefore, first of all, we recalculate the CT-EoS with experimental $C_{P}(0, T)$ data instead of the theoretical values of Powell and Fletcher (1965). The result gives a $\gamma$ value that shows not only volume but also temperature dependence, in contrast to the original result of Spetzler et al. (1972). At high temperatures, the specific heat at constant volume $\left(C_{V}\right)$ in the CT-EoS is smaller than that obtained using a harmonic approximation where the difference exceeds the expected tolerance. These inconsistencies clearly suggest the limitation of harmonic or quasiharmonic approximation. Thus, we consider introducing intrinsic anharmonicity.

The importance of intrinsic anharmonicity to thermodynamic properties of $\mathrm{NaCl}$ or similar materials such as $\mathrm{MgO}$ is already recognized (e.g., Leadbetter et al. 1969; Cowley 1971; Stacey and Isaak 2003; Oganov and Dorogokupets
2003, 2004). Others have recently proposed $\mathrm{NaCl}$ EoSs including intrinsic anharmonicity (e.g., Dorogokupets 2002; Dorogokupets and Dewaele 2007). These papers treat the intrinsic anharmonic effect on $C_{V}$ as a linear function of temperature, which is the simplest approximation neglecting higher orders in series expansion of the anharmonic Helmholtz free energy. However, these EoSs commonly fail to reproduce $\gamma$ in spite of fair reproduction of $C_{V}$ or $C_{P}$.

Nextly, we present a new thermodynamic model for $C_{V}$ and $\gamma$ that takes account of the intrinsic anharmonic effect. First, we consider positive quartic anharmonicity under an isochoric condition. Classical statistical mechanics is directly used instead of the series approximation (Leadbetter et al. 1969; Oganov and Dorogokupets 2003, 2004; Dorogokupets 2002; Dorogokupets and Dewaele 2007), which inevitably causes uncertainty by neglecting higherorder terms. We express the temperature dependence of $C_{V}$ as a function of absolute and anharmonic characteristic temperatures. Also, we take into account the ordinary harmonic quantum effect in the procedure. Second, we derive a useful equation for the temperature dependence of $\gamma$ as a thermodynamic identity. Combining these two concepts, we can calculate thermal pressure $\left(\int \gamma C_{V} / V \mathrm{~d} T\right)$ in $V-T$ space for almost all purposes.

In the anharmonic model presented here, the anharmonic characteristic temperature and its volume dependence are new parameters. Although our model rests on simple assumptions, the resulting EoS is consistent with experimental properties.

Lastly, we discuss the difference and advantages of our anharmonic model compared with the previous series approximation models.

\section{CT-EOS as experimental reference data}

We calculate the CT-EoS from the ultrasonic data of Spetzler et al. (1972), $v_{i}(0,300 \mathrm{~K})$, and $F_{i}(P, T)$, in combination with $\alpha(0, T)$ and $C_{P}(0, T)$. Before calculating the CTEoS, we fit each experimental $\alpha(0, T)$ and $C_{P}(0, T)$ value to the empirical formula

$M(T)=m_{1}\left[1+\left(T / m_{2}\right)^{-m_{3}}-m_{4} \operatorname{Exp}\left(T / m_{5}\right)\right]^{-m_{6}}$,

where $T$ is the absolute temperature. For the $\alpha(0, T)$ curve, prior to fitting, the measurement data (Rubin et al. 1961; Yates and Panter 1962; Enck and Dommel 1965; Meincke and Graham 1965; White 1965; Leadbetter and Newsham 1969; Pathak and Vasavada 1970; Kirby et al. 1972; Rapp and Merchant 1973; Legge et al. 1979; Spinolo et al. 1979; Ming et al. 1983), e.g., $(\mathrm{d} L / \mathrm{d} T)_{P} / L_{293 \mathrm{~K}}$, have been corrected with the thermodynamic definition, 
Table $1 m_{i}$ of Eq. 1 for the volume thermal expansion coefficient at zero pressure, $\alpha(0, T)$, and the isobaric specific heat at zero pressure, $C_{P}(0, T)$

\begin{tabular}{lll}
\hline$i$ & $m_{i}$ \\
\cline { 2 - 3 } & $\alpha(0, T)$ in $\left(\mathrm{K}^{-1}\right)$ & $C_{P}(0, T)$ in $\left(\mathrm{JK}^{-1} \mathrm{~mol}^{-1}\right)$ \\
\hline 1 & $2.49085 \times 10^{-5}$ & 44.4295 \\
2 & 38.7213 & 44.0448 \\
3 & 2.45015 & 2.00318 \\
4 & 0.610481 & 0.0589528 \\
5 & 4913.64 & 940.169 \\
6 & 1.51746 & 1.99351 \\
\hline
\end{tabular}

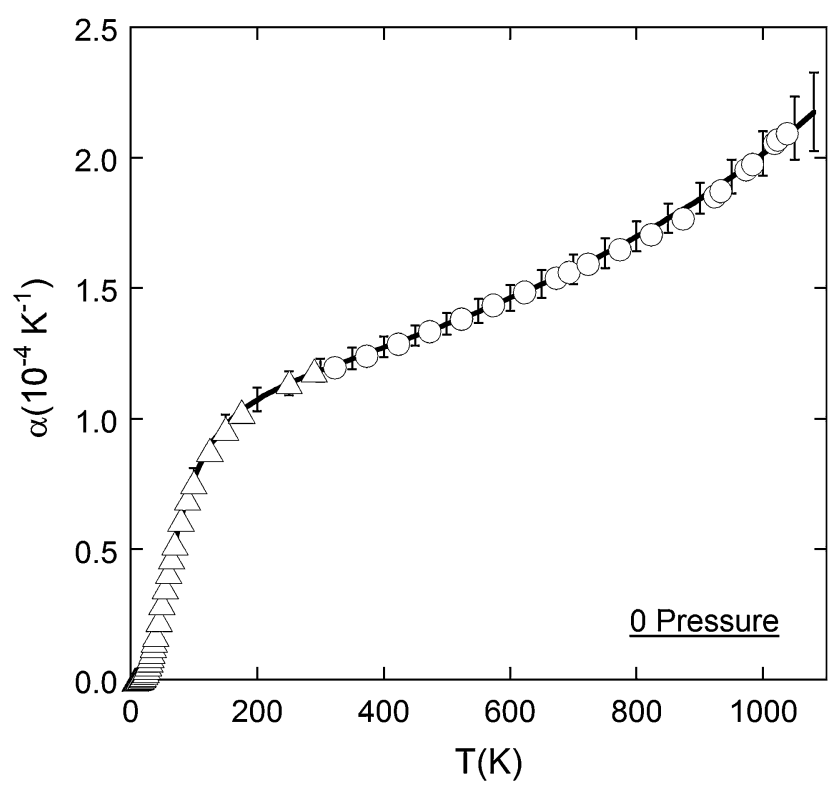

Fig. 2 Fitting line (solid line calculated from Eq. (1) and Table 1) and experimental data (circles and triangles) for the volume thermal expansion coefficient at zero pressure. Error bars shown are 15 times greater than the errors calculated from the variance-covariance matrices $\left(\varepsilon_{i j}\right.$ given in Online Resource 1). Circles Enck and Dommel (1965); triangles Meincke and Graham (1965)

$\alpha \equiv(\partial V / \partial T)_{P} / V$. Fitting $C_{P}(0, T)$ requires the $C_{P}$ (Leadbetter and Settatree 1969) and enthalpy (Magnus 1913; Roth and Bertram 1929; Dawson et al. 1963; Holm and Grønvold 1973; Archer 1997) data to be used simultaneously. Table 1 shows both fitting results. We give the corresponding calculated variance-covariance matrices $\left(\varepsilon_{i j}\right)$ in Online Resource 1. Figures 2 and 3 show the fitted curves for $\alpha(0, T)$ and $C_{P}(0, T)$, respectively, with typical experimental data (Enck and Dommel 1965; Meincke and Graham 1965; Archer 1997; Chase 1998). These figures show that Eq. 1 successfully reproduces experimental $\alpha(0, T)$ and $C_{P}(0, T)$ values with high accuracy up to the melting temperature.

Figure 4 shows the flowchart of the CT-EoS calculation, together with useful thermodynamic relations. Although

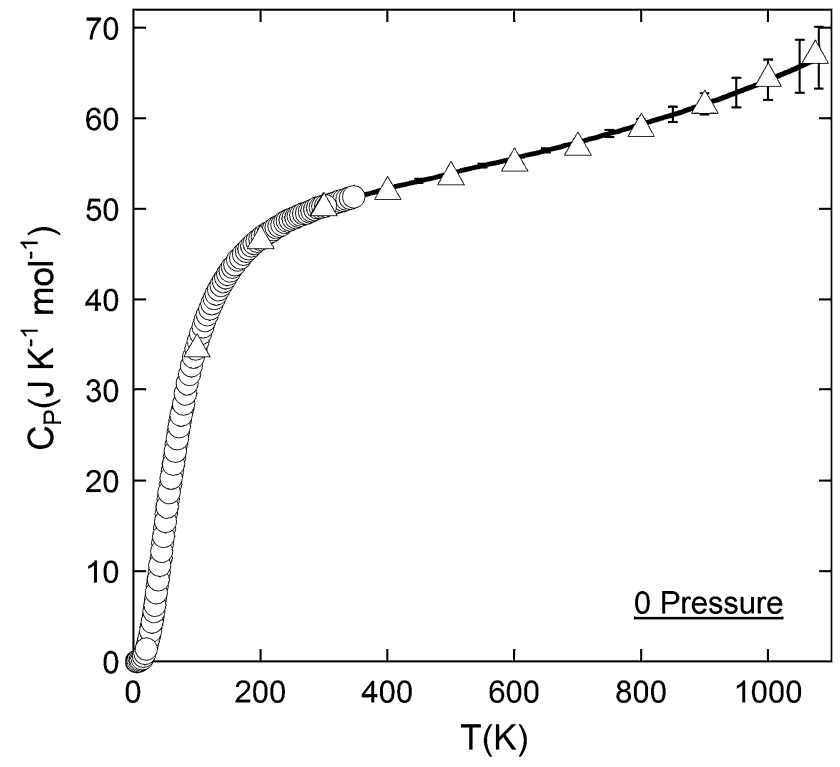

Fig. 3 Fitting line (solid line calculated from Eq. (1) and Table 1) and experimental data (circles and triangles) for the isobaric specific heat at zero pressure. Error bars shown are 10 times greater than the errors calculated from the variance-covariance matrices $\left(\varepsilon_{i j}\right.$ given in Online Resource 1). Circles Archer (1997); triangles Chase (1998)

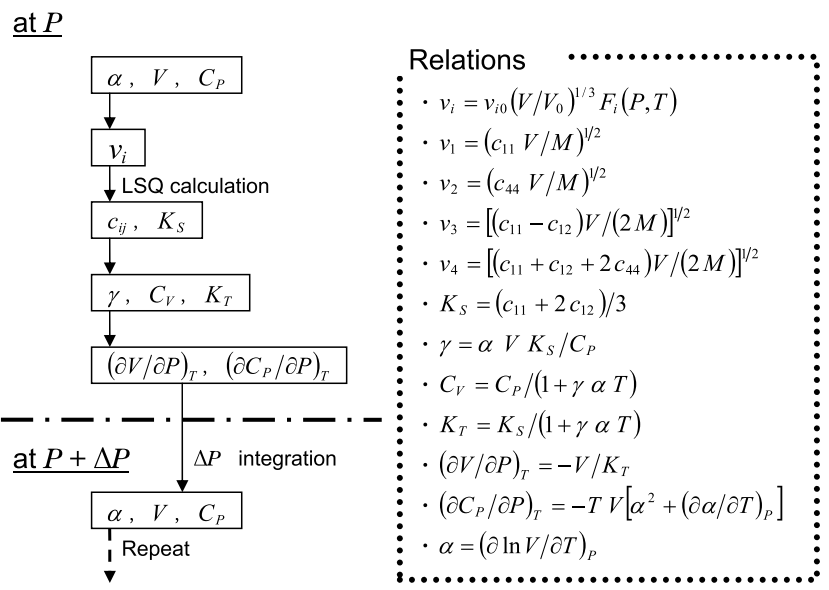

Fig. 4 Calculation flow and relationships between sound velocities and thermodynamic properties. LSQ is the least squares method

Spetzler and Yoneda (1993) assumed an elastically isotropic material for simplicity in their test analysis, we expand our calculation to treat an elastically anisotropic cubic symmetry with three independent elastic constants $\left(c_{11}, c_{12}\right.$, and $c_{44}$ ). Since Spetzler et al. (1972) have measured four individual velocities for three elastic constants, we determine the elastic constants using the least squares method (LSQ). We use the Runge-Kutta-Gill method to integrate $(\partial V / \partial P)_{T}$ and $\left(\partial C_{P} / \partial P\right)_{T}$ with respect to pressure. The pressure interval $(\Delta P)$ is set at 1 or $10 \mathrm{MPa}$ for pressure integration; the resultant CT-EoS is not significantly 
Table 2 Molar volume, $V$, in the CT-EoS (this study) and estimated tolerances (in parentheses)

Values are in $\left(10^{-5} \mathrm{~m}^{3} \mathrm{~mol}^{-1}\right)$

Table 3 Isothermal bulk modulus, $K_{T}$, in the CT-EoS (this study) and estimated tolerances (in parentheses)

Values are in $\left(10^{10} \mathrm{P}_{\mathrm{a}}\right)$

Table 4 Specific heat at constant volume, $C_{V}$, in the CT-EoS (this study) and estimated tolerances (in parentheses)

Values are in $\left(\mathrm{JK}^{-1} \mathrm{~mol}^{-1}\right)$

Table 5 Grüneisen parameter, $\gamma$, in the CT-EoS (this study) and estimated tolerances (in parentheses)

Values are non-dimensional

\begin{tabular}{lllllll}
\hline P/GP & $\mathrm{T} / \mathrm{K}$ & \multicolumn{6}{l}{} & & \\
\cline { 2 - 6 } & 300 & 400 & 500 & 600 & 700 & 800 \\
\hline 0 & 2.7016 & 2.7350 & 2.7713 & 2.8107 & 2.8537 & 2.9007 \\
& $(0.0007)$ & $(0.0014)$ & $(0.0024)$ & $(0.0037)$ & $(0.0052)$ & $(0.0070)$ \\
0.4 & 2.6583 & 2.6882 & 2.7206 & 2.7554 & 2.7929 & 2.8335 \\
& $(0.0007)$ & $(0.0012)$ & $(0.0021)$ & $(0.0032)$ & $(0.0045)$ & $(0.0060)$ \\
0.8 & 2.6188 & 2.6460 & 2.6751 & 2.7063 & 2.7397 & 2.7755 \\
& $(0.0008)$ & $(0.0011)$ & $(0.0018)$ & $(0.0028)$ & $(0.0039)$ & $(0.0052)$
\end{tabular}

\begin{tabular}{lllllll}
\hline P/GP & $\mathrm{T} / \mathrm{K}$ & \multicolumn{7}{l}{} & & \\
\cline { 2 - 6 } & 300 & 400 & 500 & 600 & 700 & 800 \\
\hline 0 & 2.3714 & 2.2122 & 2.0546 & 1.8985 & 1.7441 & 1.5917 \\
& $(0.0151)$ & $(0.0150)$ & $(0.0160)$ & $(0.0188)$ & $(0.0215)$ & $(0.0222)$ \\
0.4 & 2.5807 & 2.4264 & 2.2736 & 2.1224 & 1.9727 & 1.8248 \\
& $(0.0159)$ & $(0.0157)$ & $(0.0165)$ & $(0.0190)$ & $(0.0218)$ & $(0.0232)$ \\
0.8 & 2.7720 & 2.6231 & 2.4758 & 2.3299 & 2.1855 & 2.0425 \\
& $(0.0166)$ & $(0.0164)$ & $(0.0169)$ & $(0.0190)$ & $(0.0219)$ & $(0.0239)$ \\
\hline
\end{tabular}

\begin{tabular}{lllllll}
\hline P/GP & \multicolumn{7}{l}{$\mathrm{T} / \mathrm{K}$} & 400 & 500 & 600 & 700 & 800 \\
\cline { 2 - 6 } & 300 & 48.326 & 48.630 & 48.732 & 48.732 & 48.670 \\
\hline 0 & 47.529 & $(0.280)$ & $(0.368)$ & $(0.477)$ & $(0.606)$ & $(0.824)$ \\
& $(0.227)$ & 48.345 & 48.681 & 48.809 & 48.832 & 48.794 \\
0.4 & 47.496 & $(0.261)$ & $(0.366)$ & $(0.479)$ & $(0.587)$ & $(0.765)$ \\
& $(0.207)$ & 48.362 & 48.729 & 48.880 & 48.923 & 48.902 \\
0.8 & 47.462 & $(0.241)$ & $(0.359)$ & $(0.481)$ & $(0.577)$ & $(0.718)$ \\
& $(0.184)$ & & & & &
\end{tabular}

\begin{tabular}{lllllll}
\hline $\mathrm{P} / \mathrm{GP}_{\mathrm{a}}$ & $\mathrm{T} / \mathrm{K}$ & \multicolumn{5}{l}{} \\
\cline { 2 - 6 } & 300 & 400 & 500 & 600 & 700 & 800 \\
\hline 0 & 1.5978 & 1.5957 & 1.5977 & 1.6015 & 1.6064 & 1.6116 \\
& $(0.0606)$ & $(0.0511)$ & $(0.0512)$ & $(0.0570)$ & $(0.0608)$ & $(0.0599)$ \\
0.4 & 1.5633 & 1.5628 & 1.5658 & 1.5706 & 1.5764 & 1.5827 \\
& $(0.0618)$ & $(0.0518)$ & $(0.0507)$ & $(0.0559)$ & $(0.0602)$ & $(0.0598)$ \\
0.8 & 1.5250 & 1.5265 & 1.5308 & 1.5368 & 1.5439 & 1.5516 \\
& $(0.0626)$ & $(0.0523)$ & $(0.0501)$ & $(0.0546)$ & $(0.0592)$ & $(0.0596)$
\end{tabular}

changed by choosing different pressure intervals. Tables 2 , 3,4 , and 5 show the resultant values for $V, K_{T}, C_{V}$, and $\gamma$, respectively. Online Resource 2 also lists the results for the other thermodynamic properties.

We have examined the CT-EoS values to identify any influences resulting from errors in the data used (Tables 2, $3,4,5$ in parentheses). Here, likely errors include those in $\alpha(0, T), C_{P}(0, T), v_{i}(0,300 \mathrm{~K})$, and $F_{i}(P, T)$. We have confirmed that the influences of other errors (e.g., lattice constant and atomic weight) are negligible. The estimated tolerance of the experimental $\alpha(0, T)$ values is 15 times greater than that of the error calculated from $\varepsilon_{i j}$ (Online Resource 1) using the error propagation law (error bars in Fig. 2). We note that the total number of experimental data for $\alpha(0, T)$ is 67 (Enck and Dommel 1965; Leadbetter and Newsham 1969; Pathak and Vasavada 1970; Kirby et al. 1972; Rapp and Merchant 1973; Spinolo et al. 1979; Ming et al. 1983) in the temperature range 300-800 K, with most 


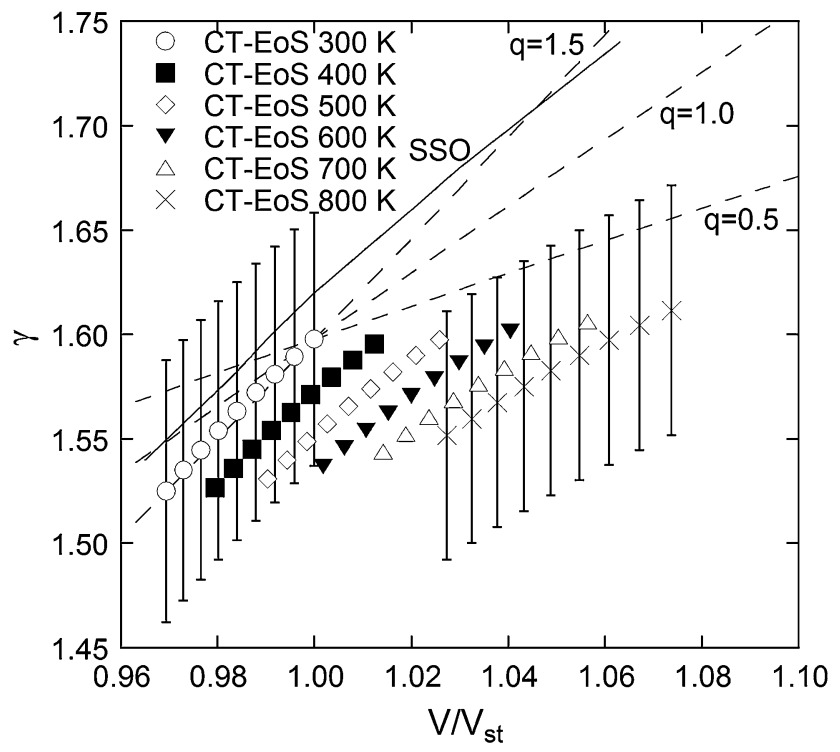

Fig. 5 Grüneisen parameter in the CT-EoS. Error bars represent tolerances for the data at 300 and $800 \mathrm{~K}$. SSO (solid line) represents the original data of Spetzler et al. (1972). Dashed lines represent ordinary power-law $\gamma$ for $q=0.5,1.0$ and 1.5. $V_{s t}$ is the molar volume under the standard conditions (zero pressure and $300 \mathrm{~K}$ )

of these values inside the tolerance $(91 \%$ or 61 out of 67 data). Similarly, the tolerance of $C_{P}(0, T)$ is estimated to be 10 times greater than the error calculated from $\varepsilon_{i j}$ (error bars in Fig. 3). The total number of experimental $C_{P}(0, T)$ and enthalpy data is 86 (Magnus 1913; Dawson et al. 1963; Leadbetter and Settatree 1969; Archer 1997) in the temperature range $300-800 \mathrm{~K}$, with most of these values also inside the tolerance ( $84 \%$ or 71 out of 86 data). As for the ultrasonic data of Spetzler et al. (1972), the tolerances for all $v_{i}(0,300 \mathrm{~K})$ values are estimated equally at $7 \mathrm{~m} / \mathrm{s}$, while the tolerances of $F_{i}(P, T)$ are calculated from the error matrices given by Spetzler et al. (1972). Thus, every thermodynamic parameter should be inside the estimated tolerances.

Figure 5 shows the resulting values for $\gamma$. The large tolerances of $\gamma$ result almost entirely from the tolerances of $\alpha(0, T)$. Although we cannot completely reject the possibility of $\gamma=\gamma(V), q=(\partial \ln \gamma / \partial \ln V)_{T} \approx 0.5 \quad$ (see dashed lines in Fig. 5) is too small compared with the other experimental estimations of 1.1-1.3 (Boehler et al. 1977; Yamamoto et al. 1987). The possibility of overestimation at low temperature and underestimation at high temperature is unlikely, because many previous studies showed nearly constant $\gamma$ at zero pressure (e.g., Leadbetter et al. 1969; Birch 1986; Yamamoto et al. 1987; Brown 1999). The corresponding curve reported by Spetzler et al. (1972) is outside the tolerance of our calculations in the larger volume regions (see SSO in Fig. 5). This discrepancy is due to the difference in the initial condition of $C_{P}(0, T)$. Our results

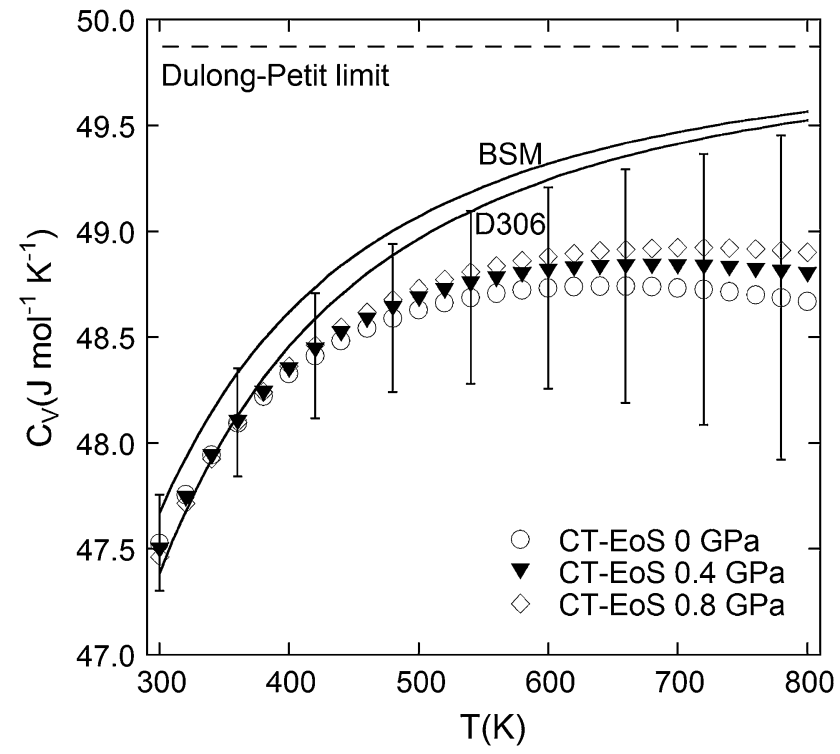

Fig. 6 Specific heat at constant volume in the CT-EoS. Error bars represent tolerances for the data at $0 \mathrm{GPa}$. D306 (lower solid curve) and BSM (upper solid curve) are calculated using the Debye model $\left(\theta_{D}=306 \mathrm{~K}\right)$ and the breathing shell model (BSM) by Nüsslein and Schröder (1967), respectively. The Dulong-Petit limit (broken line) is $49.89 \mathrm{JK}^{-1} \mathrm{~mol}^{-1}$

show not only volume dependence, but also negative temperature dependence.

Figure 6 shows the resulting $C_{V}$ value. The tolerances of $\alpha(0, T)$ and $C_{P}(0, T)$ constitute nearly all of the tolerance of $C_{V}$ and contribute equally. At higher temperature (>600 K), the $C_{V}(0, T)$ values are saturated and obviously smaller than the Dulong-Petit limit. The $C_{V}$ value obtained from the Debye model is also shown for comparison (D306 in Fig. 6). In the Debye model, the Debye temperature $\left(\theta_{D}\right)$ is estimated from the $v_{i}(0,300 \mathrm{~K})$ values of Spetzler et al. (1972) to be $306 \mathrm{~K}$. In the higher temperature region, the $C_{V}(0, T)$ value in the CT-EoS is smaller than that obtained using the Debye model beyond the tolerance. The Debye model assumes both the functional form of a lattice-vibrational spectrum (phonon spectrum) and the harmonic approximation. The inconsistency between the CTEoS and the Debye model indicates that either (or both) of these two assumptions is (or are) unsuitable for $\mathrm{NaCl}$. For a more realistic harmonic approximation model, $C_{V}$ is calculated using the phonon spectrum obtained from the breathing shell model (BSM) (Nüsslein and Schröder 1967). As shown in Fig. 6, the similarity between the $C_{V}$ values generated by the Debye model and the BSM implies that a difference in the phonon spectrum has little influence on the calculation of $C_{V}$. The difference in $C_{V}$ between the CTEoS and harmonic approximation models suggests that the harmonic approximation is insufficient, i.e., $\mathrm{NaCl}$ exhibits clear intrinsic anharmonicity. Furthermore, as shown in 
Fig. 6, the pressure dependence of $C_{V},\left(\partial C_{V} / \partial P\right)_{T}$, in the CT-EoS is likely to have a positive sign, which is in contrast to the negative sign expected when the quasi-harmonic approximation is used.

\section{Anharmonic model}

Recently, Cuccoli et al. (1990) and Rössler and Page (1995) investigated the $C_{V}$ values for an anharmonic atomic chain. The corresponding results are qualitatively consistent with each other. Here, high-temperature behavior is characterized by the $C_{V}$ values obtained from the classical anharmonic system, while low-temperature behavior is characterized by the quantum effect in the harmonic approximation. Thus, in this paper, we use a classical statistical-mechanical model for the anharmonic potential (harmonic term plus quartic term) and the quantum effect in the harmonic approximation and apply them to $C_{V}$ for an anharmonic crystal. A model for the Grüneisen parameter $(\gamma)$ is derived from the classical anharmonic $C_{V}$ model and the thermodynamic identity based on thermal pressure:

$\left(\frac{\partial C_{V}}{\partial \ln V}\right)_{T}=C_{V}\left(\frac{\partial \gamma}{\partial \ln T}\right)_{V}+\gamma\left(\frac{\partial C_{V}}{\partial \ln T}\right)_{V}$.

The Appendix gives the derivation of Eq. 2.

Specific heat from classical statistical mechanics

Here we use classical statistical mechanics to calculate $C_{V}$ for the classical anharmonic system. We assume that the $i$ th classical vibration mode follows the potential $E_{p i}\left(x_{i}\right)\left(x_{i}\right.$ is the generalized coordinates), so that the average potential energy is given by

$\bar{E}_{p i}=\frac{\int_{-\infty}^{\infty} E_{p i}\left(x_{i}\right) \exp \left[-E_{p i}\left(x_{i}\right) /\left(k_{B} T\right)\right] \mathrm{d} x_{i}}{\int_{-\infty}^{\infty} \exp \left[-E_{p i}\left(x_{i}\right) /\left(k_{B} T\right)\right] \mathrm{d} x_{i}}$,

where $k_{B}$ is Boltzmann's constant. Differentiating Eq. 3 with respect to $T$ gives the specific heat for $E_{p i}\left(x_{i}\right)$ :

$$
\begin{aligned}
C_{V p i}= & k_{B}\left(\frac{\int_{-\infty}^{\infty} E_{p i}^{2}\left(x_{i}\right) \exp \left[-E_{p i}\left(x_{i}\right) /\left(k_{B} T\right)\right] d x_{i}}{\left(k_{B} T\right)^{2} \int_{-\infty}^{\infty} \exp \left[-E_{p i}\left(x_{i}\right) /\left(k_{B} T\right)\right] d x_{i}}\right. \\
& -\left\{\frac{\int_{-\infty}^{\infty} E_{p i}\left(x_{i}\right) \exp \left[-E_{p i}\left(x_{i}\right) /\left(k_{B} T\right)\right] \mathrm{d} x_{i}}{k_{B} T \int_{-\infty}^{\infty} \exp \left[-E_{p i}\left(x_{i}\right) /\left(k_{B} T\right)\right] \mathrm{d} x_{i}}\right\} .
\end{aligned}
$$

The harmonic approximation gives $C_{V p i}=k_{B} / 2$. For the positive even $l$ th-power potential, the specific heat is $C_{V p i}=k_{B} / l$. The specific heat of the kinetic energy for the $i$ th mode is always given by $k_{B} / 2$, which can be calculated by substituting $m \dot{x}_{i}^{2} / 2$ and d $\dot{x}_{i}$ for $E_{p i}\left(x_{i}\right)$ and $d x_{i}$ in Eq. 4 , respectively.

During an isochoric temperature change, all average atomic positions are fixed geometrically in $\mathrm{B} 1(\mathrm{NaCl})$ structure. This is also expected in many other cubic structures (e.g. A1 (fcc), A2 (bcc), A4 (diamond), B2 ( $\mathrm{CsCl}), \mathrm{B} 3$ (Zincblende), etc.). On the other hand, odd terms in $E_{p i}\left(x_{i}\right)$ cause the change of the average atomic position from the geometrically fixed position. Therefore, it is obvious that the fourth-order is the smallest order of anharmonic terms, and we examine the following case to calculate the anharmonic heat capacity:

$E_{p i}\left(x_{i}\right)=k_{i} x_{i}^{2} / 2+s_{i} x_{i}^{4} / 4 \quad\left(k_{i}>0, s_{i}>0\right)$.

Conversion from $k_{i} x_{i}^{2} /\left(k_{B} T\right)$ to $x_{a \_i}^{2}$ (i.e., $x_{i}^{2}=k_{B} T x_{a \_i}^{2}$ $/ k_{i}$ ) gives

$$
\begin{aligned}
& C_{V p i \_a}\left(\sigma_{i}\right)= \\
& k_{B}\left(\frac{\int_{-\infty}^{\infty}\left(x_{a_{-} i}^{2} / 2+\sigma_{i} x_{a_{-} i}^{4} / 4\right)^{2} \exp \left[-\left(x_{a_{i} i}^{2} / 2+\sigma_{i} x_{a_{-i}}^{4} / 4\right)\right] \mathrm{d} x_{a_{-} i}}{\int_{-\infty}^{\infty} \exp \left[-\left(x_{a_{-} i}^{2} / 2+\sigma_{i} x_{a_{-} i}^{4} / 4\right)\right] \mathrm{d} x_{a_{-} i}}\right. \\
& \left.-\left\{\frac{\int_{-\infty}^{\infty}\left(x_{a_{-}}^{2} / 2+\sigma_{i} x_{a_{-} i}^{4} / 4\right) \exp \left[-\left(x_{a_{-} i}^{2} / 2+\sigma_{i} x_{a_{a} i}^{4} / 4\right)\right] \mathrm{d} x_{a_{-} i}}{\int_{-\infty}^{\infty} \exp \left[-\left(x_{a_{-} i}^{2} / 2+\sigma_{i} x_{a_{-} i}^{4} / 4\right)\right] \mathrm{d} x_{a_{-} i}}\right\}^{2}\right),
\end{aligned}
$$

where $\sigma_{i}\left(=s_{i} k_{B} T / k_{i}^{2}\right)$ is the intensity parameter of anharmonicity. Figure 7 shows the relationship between $\sigma_{i}$ and $C_{V p i \_a}$, where $C_{V p i \_a}$ equals $k_{B} / 2$ at $\sigma_{i}=0$. For

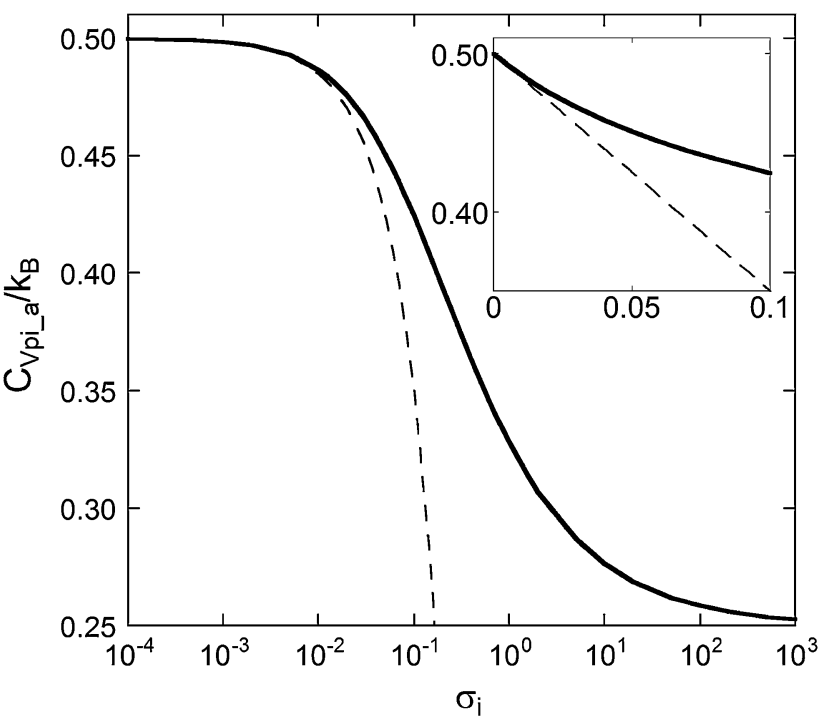

Fig. 7 Specific heat of the potential energy for a classical anharmonic solid. Assumed potential includes positive quartic term (Eq. 5). $\sigma_{i}=T / \theta_{a_{i} i}$. Solid lines are calculated from Eq. 6. Broken lines correspond to previous linear anharmonic model (Eqs. 17, 18). Horizontal axes of outer and inner figures are in logarithmic and linear scales, respectively 
larger $\sigma_{i}$ values, $C_{V p i \_a}$ approaches $k_{B} / 4$ as a lower limit. We define the anharmonic characteristic temperature as $\theta_{a_{-} i} \equiv k_{i}^{2} /\left(s_{i} k_{B}\right)=T / \sigma_{i}$. The concept of this characteristic temperature $\theta_{a_{-} i}$ is different from the anharmonicitycorrected characteristic temperature of the quasi-harmonic approximation (Holzapfel 2002; Ponkratz and Holzapfel 2004). At constant temperatures, the decrease in $\theta_{a_{-} i}$ causes an increase in anharmonicity, with a subsequent decrease in $C_{V p i \_a}$. We note that the vibration approaches a harmonic as $\theta_{a_{i} i}$ increases. To ignore the mode dependence of $\theta_{a_{-} i}$, we assume a uniform energy distribution for each of the harmonic terms and the anharmonic quartic terms in $E_{p i}\left(x_{i}\right)$ (Eq. 5). Consequently, we express the specific heat $C_{V a}$ of a classical anharmonic solid as a function of $T / \theta_{a}$ :

$C_{V a}\left(\frac{T}{\theta_{a}}\right)=3 n N_{A}\left[\frac{k_{B}}{2}+C_{V p i_{-} a}\left(\frac{T}{\theta_{a}}\right)\right]$,

where $n$ refers to the number of atoms in the chemical formula and $N_{A}$ is Avogadro's number.

Temperature dependence of Grüneisen parameter

The temperature dependence of $\gamma$ is related to the differential coefficients of $C_{V}$ by Eq. 2. Using $C_{V}=C_{V a}\left[T / \theta_{a}(V)\right]$ (Eq. 7) and $\gamma_{a}=-\left(\partial \ln \theta_{a} / \partial \ln V\right)_{T}$, we have

$\left(\frac{\partial C_{V}}{\partial \ln V}\right)_{T}=\gamma_{a} \frac{T}{\theta_{a}} C_{V a}^{\prime}$

and

$\left(\frac{\partial C_{V}}{\partial \ln T}\right)_{V}=\frac{T}{\theta_{a}} C_{V a}^{\prime}$.

Substituting the above two equations into Eq. 2, we have

$\left(\frac{\partial \gamma}{\partial \ln T}\right)_{V}=\left(\gamma_{a}-\gamma\right) \frac{T}{\theta_{a}} \frac{C_{V a}^{\prime}}{C_{V a}}$.

Assuming $\theta_{a}$ is a function of only $V, \gamma_{a}$ is constant or independent of $T$ at a given $V$. Equation 10 can be solved analytically to obtain

$\gamma(T)=\gamma_{a}+\frac{3 n R\left(\gamma_{h}-\gamma_{a}\right)}{C_{V a}\left(T / \theta_{a}\right)}$,

where $\gamma_{h}=\gamma(V, 0 \mathrm{~K})$ is the harmonic Grüneisen parameter and $R$ is the gas constant. Incidentally, Eq. 11 is equivalent to Eq. (2.3) in Leadbetter (1968), $\gamma C_{V}=\gamma^{\mathrm{qh}} C_{V}^{\mathrm{qh}}+\gamma^{\mathrm{anh}} \Delta C_{V}^{\mathrm{anh}}$.

Modeling of thermal pressure

We use Eq. 11 to model $\gamma$ as a function of $V$ and $T$. For simplicity, in the case of $\mathrm{NaCl}$, we assume a constant $\gamma_{a}$ regardless of $V$ and an ordinary power-law volume-dependent $\gamma_{h}$. Hence,
$\gamma(V, T)=\gamma_{a}+6 R \frac{\gamma_{h}(V)-\gamma_{a}}{C_{V a}\left[T / \theta_{a}(V)\right]}$

with

$\gamma_{h}(V)=\gamma_{h_{-} s t}\left(V / V_{s t}\right)^{q_{h}}$,

and

$\theta_{a}(V)=\theta_{a_{-} s t}\left(V / V_{s t}\right)^{-\gamma a}$,

where the subscript "st" refers to the standard conditions (zero pressure and $300 \mathrm{~K}$ ). We confirm the validity of this simplification later by comparing this anharmonic model with the CT-EoS.

Here, we write the molar volume at $0 \mathrm{~K}$ and zero pressure as $V_{0}$. We model the specific heat $C_{V}\left(V_{0}, T\right)$ as the product of $C_{V a}\left(T / \theta_{a}\right)$ (Eq. 7) and the quasi-harmonic quantum correction,

$$
\begin{aligned}
C_{V}\left(V_{0}, T\right)= & C_{V a}\left[\frac{T}{\theta_{a}\left(V_{0}\right)}\right] \frac{1}{6 N_{A}} \int_{0}^{\omega_{\operatorname{Max}}} \\
& \frac{\left[\hbar \omega /\left(k_{B} T\right)\right]^{2} \exp \left[\hbar \omega /\left(k_{B} T\right)\right]}{\left(\exp \left[\hbar \omega /\left(k_{B} T\right)\right]-1\right)^{2}} g(\omega) \mathrm{d} \omega,
\end{aligned}
$$

where $\omega$ is the angular frequency, $\hbar$ is Planck's constant divided by $2 \pi$, and $g(\omega)$ is the frequency distribution function from BSM (Nüsslein and Schröder 1967).

Using the models for $\gamma(V, T)$ (Eqs. 12, 13, 14) and $C_{V}\left(V_{0}, T\right)$ (Eq. 15), $C_{V}(V, T)$ can be derived by integrating Eq. 2 with respect to $\ln V$. Hence, we obtain the thermal pressure $\left(\int_{0}^{T} \gamma C_{V} V^{-1} \mathrm{~d} T\right)$ as a function of $V$ and $T$. Additional knowledge of the compression curve, e.g., the BirchMurnaghan EoS (Murnaghan 1944; Birch 1952), Vinet EoS (Vinet et al. 1987), or pseudospinodal EoS (Baonza et al. 1995; Taravillo et al. 2002), at a certain temperature (e.g. $300 \mathrm{~K})$ complements the total EoS.

\section{Comparing with the CT-EoS}

We use the CT-EoS results to execute the LSQ calculation for the anharmonic model using the CT-EoS results (Tables 2, 3, 4, 5). The resultant model parameters are $\gamma_{h_{s} s t}=1.622 \pm 0.025, q_{h}=1.35 \pm 0.57$, $\theta_{a_{s} s t}=(86 \pm 12) \times 10^{3} \mathrm{~K}$, and $\gamma_{a}=9.5 \pm 3.3$. Figure 8 compares the $\gamma$ values obtained from the anharmonic model and the CT-EoS. Here, both the temperature and volume dependences of the anharmonic model are consistent with those of the CT-EoS, within an allowable tolerance, so that our anharmonic model is more desirable than the frequently used $\gamma=\gamma(V)$ model. Figure 9 compares the $C_{V}$ value of the anharmonic model with that of the CT-EoS. Though the volume changes in $C_{V}$ have to be computed by 
integrating Eq. 2 using the models of $\gamma(V, T)$ and $C_{V}\left(V_{0}, T\right)$, $C_{V}$ in this study is easily calculated from Eq. 15 , in which $V$ is substituted for $V_{0}$ and

$$
\begin{aligned}
g(V, \omega)= & \exp \left\{\frac{\gamma_{h}}{q}\left[\left(\frac{V}{V_{s t}}\right)^{q}-\left(\frac{V_{0}}{V_{s t}}\right)^{q}\right]\right\} \\
& g\left(\omega / \exp \left\{\frac{\gamma_{h}}{q}\left[\left(\frac{V_{0}}{V_{s t}}\right)^{q}-\left(\frac{V}{V_{s t}}\right)^{q}\right]\right\}\right)
\end{aligned}
$$

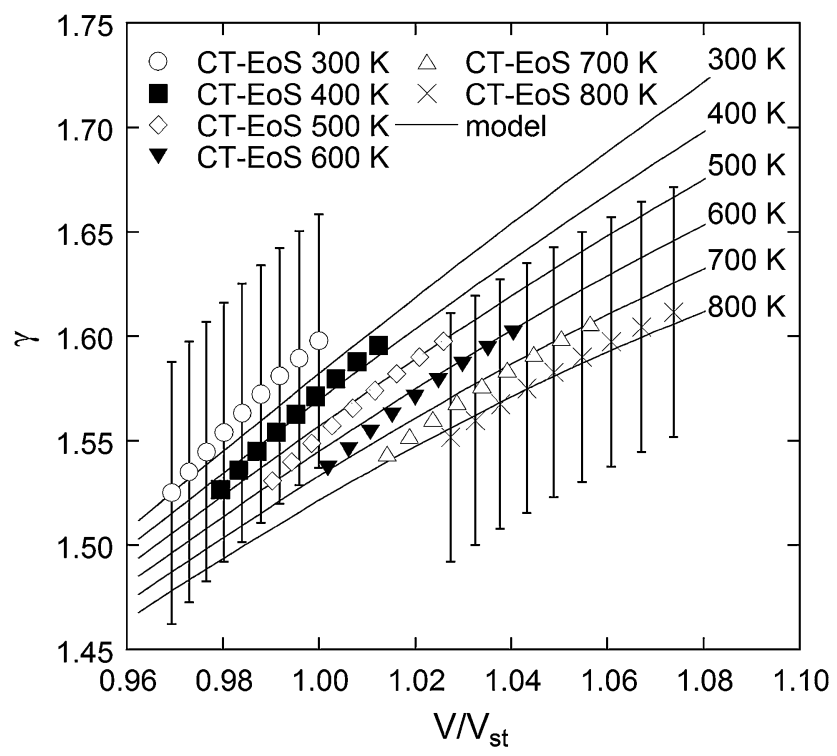

Fig. 8 Grüneisen parameter calculated using the anharmonic model (solid lines). The CT-EoS data used are the same as those in Fig. 5

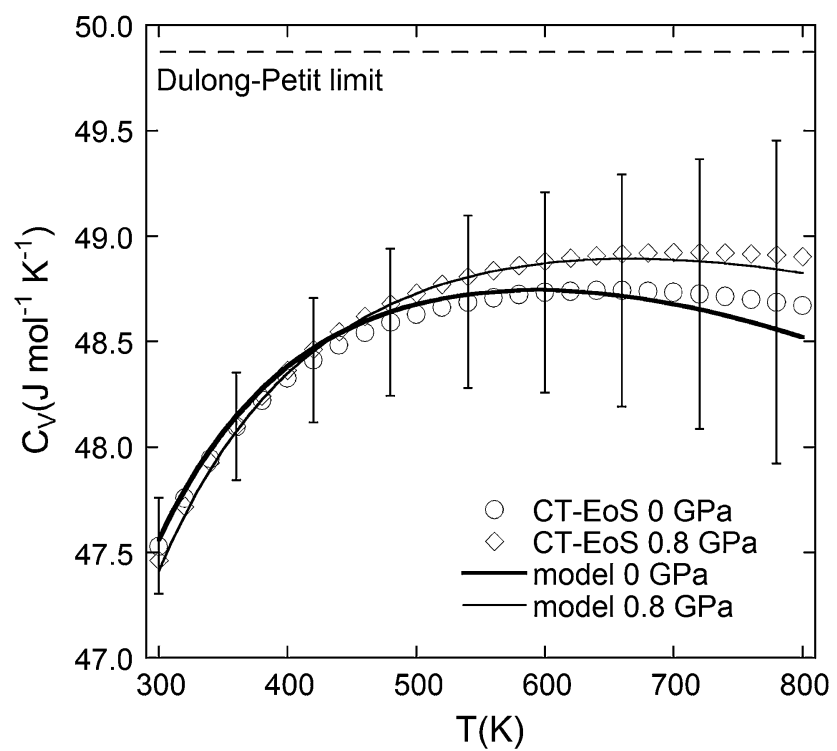

Fig. 9 Specific heat calculated using the anharmonic model (bold line $0 \mathrm{GPa}$, thin line $0.8 \mathrm{GPa}$ ). The CT-EoS data used are the same as those in Fig. 6 is substituted for $g(\omega)$. In this calculation, we use the data for $V$ in Table 2. The calculated $C_{V}$ value is consistent with that of the CT-EoS, within an allowable tolerance. These results demonstrate that our modeling and parameterization are sufficient.

\section{Discussion}

Our recalculation of the CT-EoS using correct $C_{P}$ data (Eq. 1 with Table 1; Fig. 3) yields very different $\gamma$ values from those of Spetzler et al. (1972) (Fig. 5). The error estimation shown in Tables 2, 3, 4, and 5 shows that our CTEoS achieves a level of accuracy that has not been achieved before. The new CT-EoS is important as a highly accurate experimental reference for physical modeling of thermoelasticity of solids. Boehler (1981) directly measured $(\partial T / \partial P)_{S}$ for the $\mathrm{NaCl}-\mathrm{B} 1$ phase up to $5 \mathrm{GPa}$ and $1,073 \mathrm{~K}$. Because $(\partial T / \partial P)_{S}=\gamma T / K_{S}$, we can compare the results with that of the CT-EoS. Figure 10 shows excellent agreement between the measured $(\partial T / \partial P)_{S}$ and $\gamma T / K_{S}$ from the present CT-EoS at 298, 473, 673, and $800 \mathrm{~K}$, which supports the reliability of the present CT-EoS.

The $K_{T}-V$ relationship (Fig. 11) is one of the most useful properties of the CT-EoS, where the temperature dependency of $K_{T}$ is essentially negligible (Birch 1986; Anderson 1999). However, extrapolation of the $K_{T}=K_{T}(V)$ hypothesis requires more detailed consideration. To confirm that $K_{T}=K_{T}(V)$, we examine the CT-EoS to determine whether $\left(\partial \ln K_{T} / \partial \ln V\right)_{T}=\left(\partial \ln K_{T} / \partial \ln V\right)_{P}$ is satisfied; that is,

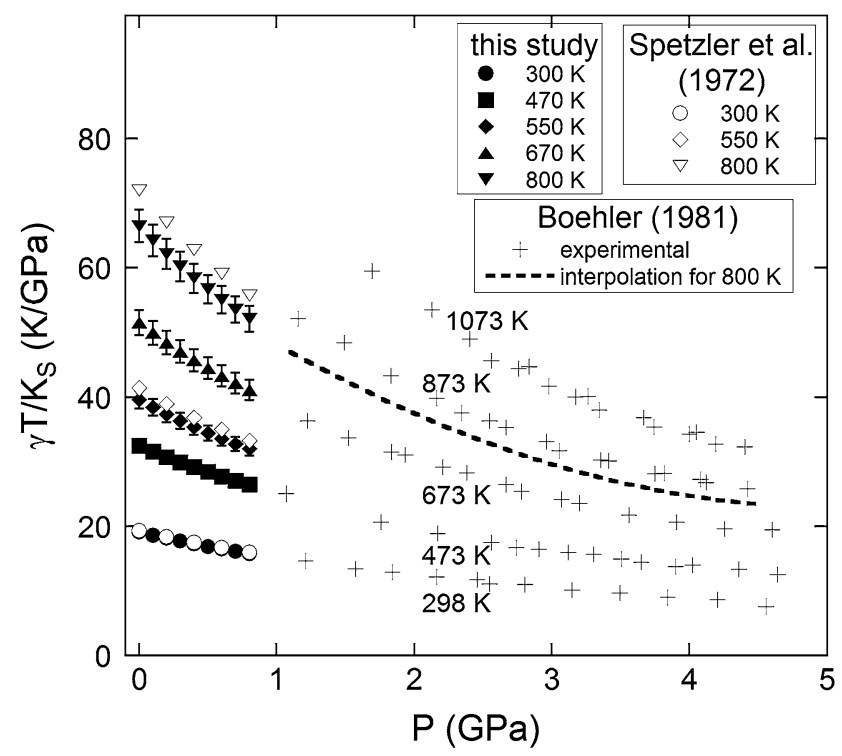

Fig. 10 Comparison of the adiabat data $\left((\partial T / \partial P)_{S}=\gamma T / K_{S}\right)$. The data sources are specified in the legend inside the plot. The broken line shows the line at $800 \mathrm{~K}$ interpolated from the original data of Boehler (1981) 


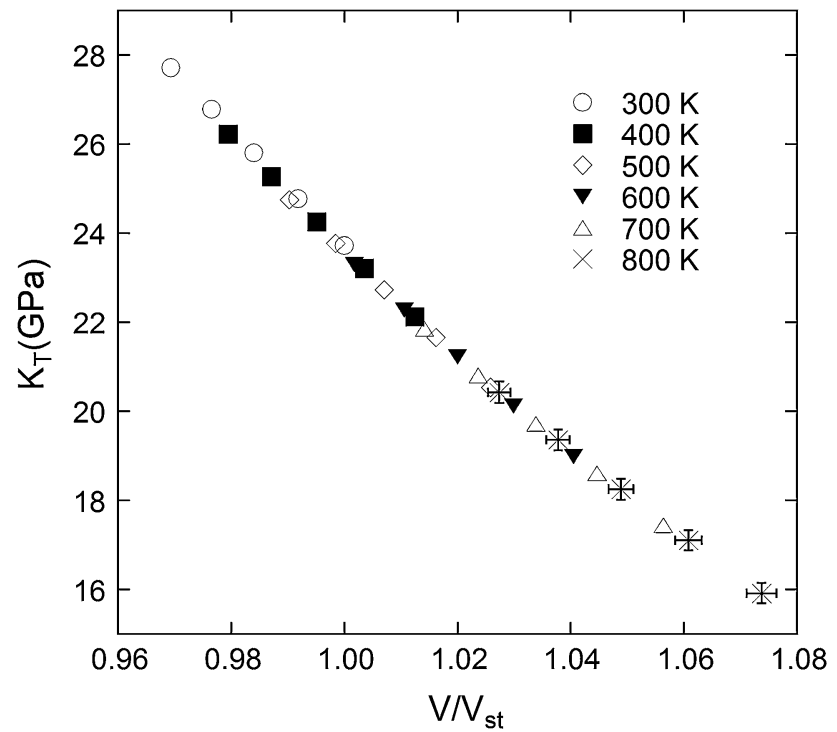

Fig. 11 Isothermal bulk modulus in the CT-EoS. Error bars represent tolerances for the data at $800 \mathrm{~K}$

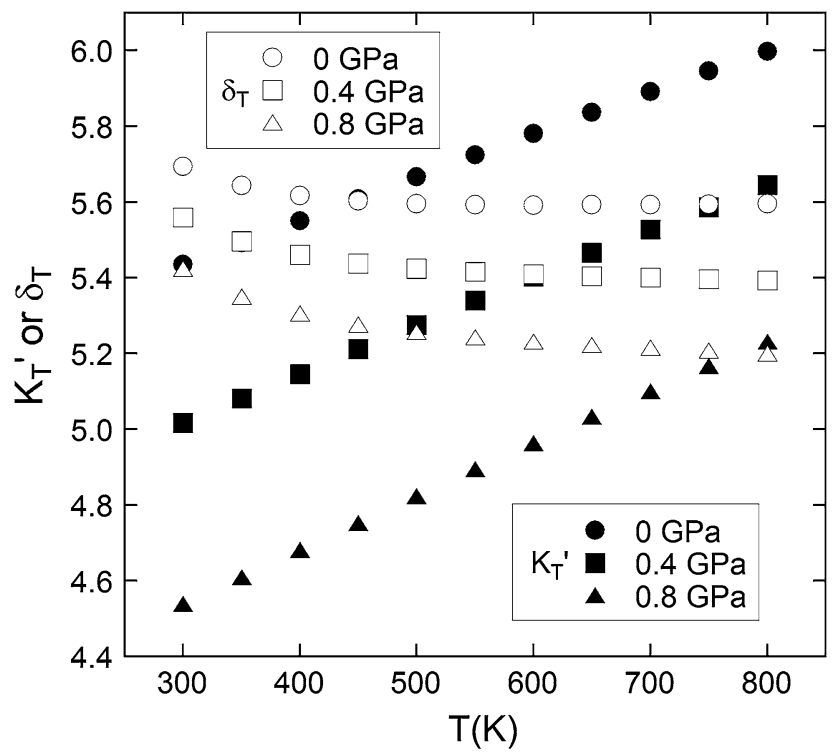

Fig. 12 Pressure derivative of the isothermal bulk modulus $\left(K_{T}^{\prime}\right)$ and the isothermal Anderson-Grüneisen parameter $\left(\delta_{T}\right)$ in the CT-EoS

$K_{T}^{\prime}=\delta_{T}\left(K_{T}^{\prime}\right.$ being the pressure derivative of $K_{T}$, and $\delta_{T}$ the isothermal Anderson-Grüneisen parameter). However, the results in Fig. 12 indicate that the CT-EoS does not satisfy $K_{T}^{\prime}=\delta_{T}$. Thus, $K_{T}=K_{T}(V)$ only holds within a limited $P-T$ range, so that an EoS based on this hypothesis cannot be supported.

To evaluate $C_{V}$ for positive quartic anharmonicity (Eq. 5), we use statistical mechanics, which enables us to avoid the imperfection of series approximation. The lowest order series approximation
Table 6 Specific heat of a classical positive quartic anharmonic solid $\left(C_{V a} / 3 n R-1\right)$

\begin{tabular}{|c|c|c|c|}
\hline$T / \theta_{a}$ & $\times 10^{-4}$ & $\times 10^{-3}$ & $\times 10^{-2}$ \\
\hline 1.0 & $\begin{array}{l}-0.000150 \\
(99.88)\end{array}$ & $\begin{array}{l}-0.001482 \\
(98.82)\end{array}$ & $\begin{array}{l}-0.013448 \\
(89.65)\end{array}$ \\
\hline 1.2 & $\begin{array}{l}-0.000180 \\
(99.85)\end{array}$ & $\begin{array}{l}-0.001775 \\
(98.59)\end{array}$ & $\begin{array}{l}-0.015822 \\
(87.90)\end{array}$ \\
\hline 1.4 & $\begin{array}{l}-0.000210 \\
(99.83)\end{array}$ & $\begin{array}{l}-0.002066 \\
(98.36)\end{array}$ & $\begin{array}{l}-0.018110 \\
(86.24)\end{array}$ \\
\hline 1.7 & $\begin{array}{l}-0.000254 \\
(99.79)\end{array}$ & $\begin{array}{l}-0.002499 \\
(98.02)\end{array}$ & $\begin{array}{l}-0.021392 \\
(83.89)\end{array}$ \\
\hline 2.0 & $\begin{array}{l}-0.000299 \\
(99.76)\end{array}$ & $\begin{array}{l}-0.002930 \\
(97.68)\end{array}$ & $\begin{array}{l}-0.024509 \\
(81.7)\end{array}$ \\
\hline 2.5 & $\begin{array}{l}-0.000374 \\
(99.70)\end{array}$ & $\begin{array}{l}-0.003642 \\
(97.12)\end{array}$ & $\begin{array}{l}-0.029377 \\
(78.34)\end{array}$ \\
\hline 3.2 & $\begin{array}{l}-0.000478 \\
(99.62)\end{array}$ & $\begin{array}{l}-0.004625 \\
(96.35)\end{array}$ & $\begin{array}{l}-0.035602 \\
(74.17)\end{array}$ \\
\hline 4.0 & $\begin{array}{l}-0.000597 \\
(99.52)\end{array}$ & $\begin{array}{l}-0.005730 \\
(95.49)\end{array}$ & $\begin{array}{l}-0.042010 \\
(70.02)\end{array}$ \\
\hline 5.0 & $\begin{array}{l}-0.000746 \\
(99.40)\end{array}$ & $\begin{array}{l}-0.007084 \\
(94.45)\end{array}$ & $\begin{array}{l}-0.049156 \\
(65.54)\end{array}$ \\
\hline 6.2 & $\begin{array}{l}-0.000923 \\
(99.26)\end{array}$ & $\begin{array}{l}-0.008671 \\
(93.24)\end{array}$ & $\begin{array}{l}-0.056715 \\
(60.98)\end{array}$ \\
\hline 8.0 & $\begin{array}{l}-0.001189 \\
(99.05)\end{array}$ & $\begin{array}{l}-0.010979 \\
(91.49)\end{array}$ & $\begin{array}{l}-0.066446 \\
(55.37)\end{array}$ \\
\hline
\end{tabular}

Values are non-dimensional. Values in parentheses are ratios (in \%) to the linear anharmonic model (Eqs. 17, 18)

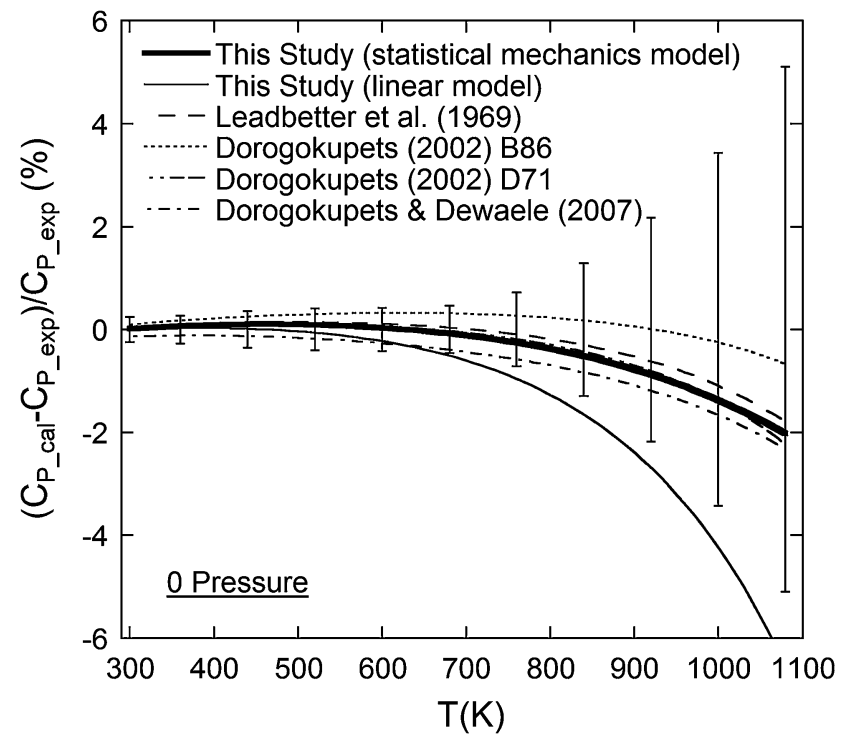

Fig. 13 Comparison of the isobaric specific heat at zero pressure. The relative ratios of model values to experimental values are shown. Experimental values are from Eq. 1 with Table 1. Bold line statisticalmechanical model from Eqs. 6 and 7; thin lines linear anharmonicity models from Eqs. 17 and 18. Parameters used are listed in Table 7. Error bars are shown for experimental values 
Table 7 Parameters for the Grüneisen parameter, $\gamma$. Previous data are converted according to the definition of this study. Values in parentheses are for $V_{s t}$

\begin{tabular}{|c|c|c|c|c|c|}
\hline \multirow[t]{2}{*}{ Parameter } & \multirow[t]{2}{*}{ This Study } & \multirow{2}{*}{$\begin{array}{l}\text { Leadbetter et al. } \\
\text { (1969) }\end{array}$} & \multicolumn{2}{|c|}{ Dorogokupets (2002) } & \multirow{2}{*}{$\begin{array}{l}\text { Dorogokupets \& } \\
\text { Dewaele (2007) }\end{array}$} \\
\hline & & & $\mathrm{B} 86$ & D71 & \\
\hline$\gamma_{h \_s t}$ & 1.622 & 1.622 & 1.608 & 1.657 & 1.64 \\
\hline$q_{h}$ & 1.35 & 1.10 & (1.034) & $(1.532)$ & $(1.382)$ \\
\hline$\theta_{a_{-} s t}\left(10^{3} \mathrm{~K}\right)$ & 86 & 84.5 & 105.0 & 73.1 & 62.4 \\
\hline$\gamma_{a}$ & 9.5 & 7 & 7.348 & 8.435 & 7.02 \\
\hline
\end{tabular}

$C_{V a} /(3 n R)=1+A T$

is the most often used at the moment. Also, for $\mathrm{NaCl}$, some researchers (Leadbetter et al. 1969; Dorogokupets 2002; Dorogokupets and Dewaele 2007) have proposed EoSs based on Eq. 17. The anharmonic parameter $A$ is related to $\theta_{a}$ in the low-temperature approximation (Kittel 1953; Oganov and Dorogokupets 2004):

$A=-3 /\left(2 \theta_{a}\right)$.

As shown in Table 6 and Fig. 7, the linear approximation Eq. 17 is good for $T / \theta_{a}<2 \times 10^{-3}$, but inadequate for $T / \theta_{a}>10^{-2}$. For $\mathrm{NaCl}$ at zero pressure, the condition $T / \theta_{a}>10^{-2}$ is matched at above $600 \mathrm{~K}$.

If our anharmonic model includes sufficient physical basis, thermoelastic properties should be estimated accurately even beyond the $P-T$ conditions in which the CT-EoS data are used for parameter optimization. Figure $13 \mathrm{com}-$ pares experimental $C_{P}$ at zero pressure with the present and previous anharmonic models (Leadbetter et al. 1969; Dorogokupets 2002; Dorogokupets and Dewaele 2007). In the anharmonic models, the values of $C_{P}$ are calculated from $C_{P}=C_{V}(1+\gamma \alpha T)$ with the values of $\alpha(0, T)$ from Eq. 1 and Table 1. Table 7 lists the parameters for the anharmonic models. Figure 13 shows that our model succeeds in estimating $C_{P}$ with high accuracy even beyond $800 \mathrm{~K}$, which is the high-temperature limit of the CT-EoS data. However, previous linear anharmonic models can also reproduce experimental $C_{P}$ with equally high accuracy. The linear anharmonic model (Eqs. 17, 18) with parameters in this study underestimates the value of $C_{P}$ above $600 \mathrm{~K}$, because the condition $T / \theta_{a}>10^{-2}$ is appropriate. Conversely, all previous models use small values of $\gamma_{a}$ (Table 7) to avoid underestimating $C_{P}$ at high temperatures. Equation $11 \mathrm{can}$ be rewritten as

$\gamma=\left(3 n R \gamma_{h}+\Delta C_{V a} \gamma_{a}\right) / C_{V a}$

with $\Delta C_{V a}=C_{V a}-3 n R$. Because the sign of $\Delta C_{V a}$ is always negative for positive quartic anharmonicity, Eq. 19 implies that a small value of $\gamma_{a}$ causes overestimation of $\gamma$ at high temperatures. Figure 14 compares experimental $\gamma$ at zero pressure to the present and previous anharmonic models (Leadbetter et al. 1969; Dorogokupets 2002; Dorogokupets and Dewaele 2007). Our model best reproduces

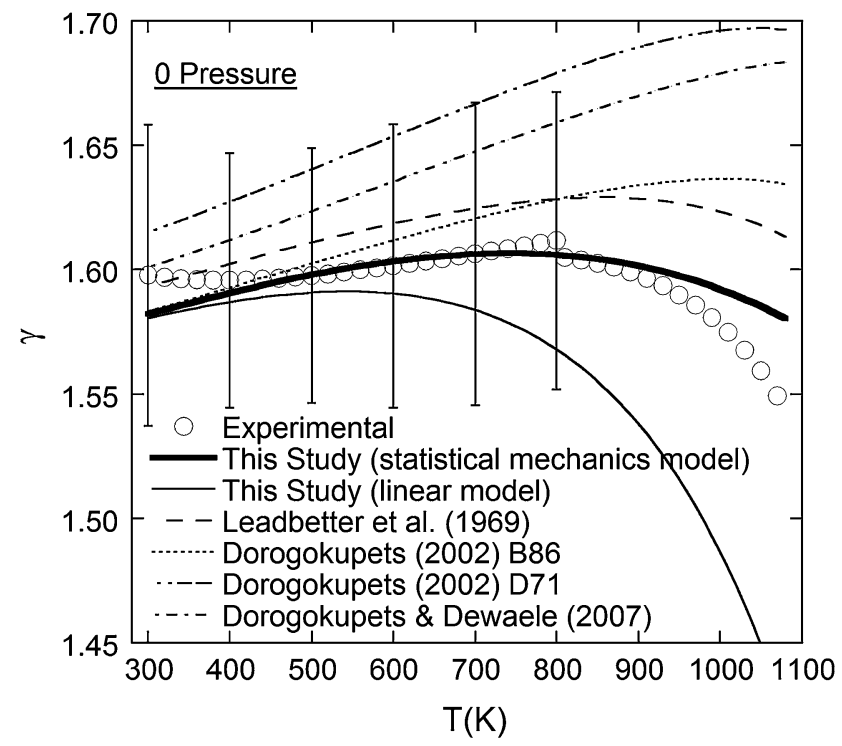

Fig. 14 Comparison of the Grüneisen parameter at zero pressure. Experimental values up to $800 \mathrm{~K}$ are from the CT-EoS results, and for over $800 \mathrm{~K}$ are calculated from $\alpha, C_{P}$ (Eq. 1 with Table 1) and $K_{S}$ (Slagle and McKinstry 1967). Bold line statistical-mechanical model from Eqs. 6, 7, and 12; thin lines linear anharmonicity models from Eqs. 12, 17, and 18. Parameters used are listed in Table 7. Error bars are shown for the CT-EoS results

the experimental values. All previous models overestimate $\gamma$, however, within the margin of error.

The good agreement between our anharmonic model and the experimental $C_{P}$ (Fig. 13), $C_{V}$ (Fig. 9) and $\gamma$ (Figs. 8, 14) shows the validity of our anharmonic model for the $\mathrm{NaCl}-\mathrm{B} 1$ phase below $1 \mathrm{GPa}$. Since $\theta_{a}$ depends on $V$ for large powers of $\gamma_{a}$ (e.g., 9.5 in this study) in Eq. 14, a slight decrease in $V$ causes a rapid increase in $\theta_{a}$ so that the anharmonicity disappears. For example, $\theta_{a}$ is nearly $1 \times 10^{6} \mathrm{~K}$ at $V / V_{s t} \approx 0.8$ (about $10 \mathrm{GPa}$ ), and therefore, $1,000 \mathrm{~K}$ gives $T / \theta_{a} \approx 10^{-3}$, at which a quasi-harmonic approximation almost holds (Table 6; Fig. 7).

The positive quartic anharmonicity reduces both $C_{V}$ and $\gamma$ values, especially at low pressure. Therefore, previous quasi-harmonic models with the power-law $\gamma$ have overestimated thermal pressure at zero pressure. Consequently, $\alpha(0, T)$ values have also been overestimated, as recognized in Decker's (1971) EoS. To reproduce $\alpha(0, T)$ values, 
Brown (1999) assumed that $\gamma$ values are constant regardless of $V$ in the expanded volume region. The present CTEoS and anharmonic model give nearly constant $\gamma$ values regardless of $V$ at zero pressure (Table 5 and Fig. 14). Thus, the anharmonic model supports Brown's (1999) hypothesis for $\gamma$ only at zero pressure.

In the preceding section, we simply calculated $C_{V}$ from Eqs. 15 and 16 to avoid numerical integration of Eq. 2. This procedure causes overestimation of $\left(\partial C_{V} / \partial \ln V\right)_{T}$. Using Eqs. 17 and 18 and the Debye heat capacity $C_{V_{-} D}$, we can write the overestimation as

$\delta\left(\partial C_{V} / \partial \ln V\right)_{T}=\frac{3 T^{2}}{2 \theta_{D} \theta_{a}}\left(\gamma_{a}-\gamma_{h}\right) C_{V_{-} D}^{\prime}$.

Because the maximum value of $C_{V_{-} D}^{\prime}$ is $3.168(3 n R)$ at $T=0.164 \theta_{D}$, the maximum error in $\left(\partial C_{V} / \partial \ln V\right)_{T}$ is $0.128\left(\gamma_{a}-\gamma_{h}\right)(3 n R)\left(\theta_{D} / \theta_{a}\right)$. For the compression from $V_{s t}$ to $0.9 V_{s t}$ in the $\mathrm{NaCl}$ case, this procedural error is less than $0.3 \%$ of $C_{V}$ and negligible.

Anharmonicity is known to cause frequency shifts in vibration modes, where these frequency shifts typically influence the quantum effect of $C_{V}$ (Schwarz 1976). Here we estimate the frequency shift in a one-particle model to roughly ascertain the magnitude of the influence. At sufficiently high temperatures $\left(T / \theta_{a} \approx 10^{-1}\right)$, the frequency is calculated to shift just $3.5 \%$ to a higher frequency. This amount is comparable to the error in the frequency distribution function and can therefore be ignored. Schwarz (1976) rigorously calculated the value of $C_{V}$ for an anharmonic oscillator with harmonic and positive quartic potential. The result (see Fig. 1 in Schwarz 1976) shows that the harmonic quantum correction is satisfactory even under a weak anharmonic condition, $\lambda \leq 0.05\left(\lambda=\theta_{E} /\left(4 \theta_{a}\right)\right.$, where $\theta_{E}$ is the Einstein characteristic temperature). Thus, our model works well under the condition $\theta_{E} \leq \theta_{a} / 5$. Note that our $\mathrm{NaCl}$ analysis satisfies the condition (maximum $\theta_{E} \approx \theta_{D}=306 \mathrm{~K}$ and $\theta_{a}>40 \times 10^{3} \mathrm{~K}$ ).

There are two bold assumptions in our anharmonic model. The first of these is uniform $\theta_{a}$ regardless of vibration mode. The second is constant $\gamma_{a}$ regardless of $V$. The large errors in $C_{V}$ and $\gamma$ (Tables 4, 5; Figs. 8, 9) make it difficult to verify our assumptions. For a stricter formulation and its evaluation, we need a precise measurement for thermal expansion $(\alpha)$, heat capacity $\left(C_{P}\right.$ or $\left.C_{V}\right)$, and vibrational spectrum $(g(\omega))$, especially at high temperatures. Also, high-pressure measurements (e.g. Murphy et al. 2011; Yoneda et al. 2009) are important. Very recently, Matsui et al. (2012) reported the simultaneous measurements of ultrasonic velocities and lattice constants of the polycrystalline $\mathrm{NaCl}-\mathrm{B} 1$ phase up to $12 \mathrm{GPa}$ and $673 \mathrm{~K}$. Extending the $P-T$ range of the CT-EoS to study intrinsic anharmonicity would require a similar experiment at higher temperatures with a narrower $P-T$ interval.
For a complicated crystal structure, average atomic positions may be affected by temperature change even under the isochoric condition. In this case, the odd term in $E_{p i}\left(x_{i}\right)$ is not negligible. Even in the 3rd-order anharmonicity of $E_{p i}\left(x_{i}\right)$, Eq. 17 is applicable as a low-temperature approximation (Kittel 1953). However, it is uncertain what the advantage of Eq. 6 as a substitute for Eq. 17 is at high temperature.

In $\mathrm{KCl}$ and $\mathrm{KBr}$ case, anharmonicity causes the heat capacity $C_{V}$ to exceed the Dulong-Petit limit (Leadbetter et al. 1969). This means that the sign of $A$ in Eq. 17 is positive; conversely, $\theta_{a}$ is negative from Eq. 18 . For negative $\theta_{a}$, the potential $E_{p i}$ shows negative divergence; therefore, the potential heat capacity Eq. 4 cannot be integrated without adding a positive even higher-order term. For the positive 6th-order term, we obtain

$E_{p i}\left(x_{i}\right)=k_{i} \frac{x_{i}^{2}}{2}+s_{i} \frac{x_{i}^{4}}{4}+t_{i} \frac{x_{i}^{6}}{6} \quad\left(k_{i}>0, s_{i}<0, t_{i}>0\right)$.

Equation 4 with Eq. 21 can be calculated by introducing the 6th-order anharmonic characteristic temperature $\theta_{a_{-} 6} \equiv\left(k_{i}^{3} /\left(t_{i} k_{B}^{2}\right)\right)^{1 / 2}$. However, it is empirical and preliminary. The relation $\theta_{a_{-6}}=-2.212 \theta_{a}$ seems to match the temperature dependence of $C_{V}$ for both $\mathrm{KCl}$ and $\mathrm{KBr}$ simultaneously (Fig. 15). Although the potential (Eq. 21) with $\theta_{a_{-} 6}=-2.212 \theta_{a}$ is obviously a triple-well potential, we cannot ascertain its physical meaning or generality at present. Thus, further study is needed for the negative $\theta_{a}$ case.

\section{Conclusion}

Recalculating the complete travel-time equation of state (CT-EoS) of the $\mathrm{NaCl}$ (B1 phase) for temperature up to $800 \mathrm{~K}$ and pressure up to $0.8 \mathrm{GPa}$ gives a highly accurate experimental reference for physical modeling of thermoelastic properties of solids. The CT-EoS yields accurate $\gamma$ and $C_{V}$ values that cannot be obtained by either the ordinary quasi-harmonic Debye or the $\gamma=\gamma(V)$ models. Carefully comparing the $C_{V}$ values of the harmonic models and CT-EoS shows that $\mathrm{NaCl}$ has clear intrinsic anharmonicity.

We introduced positive quartic anharmonicity to explain the temperature and pressure dependences of $\gamma$ and $C_{V}$ in the CT-EoS. Here, we used classical statistical mechanics for the anharmonic potential and quantum effects in a harmonic approximation and applied them to the $C_{V}$ model of an anharmonic crystal (Eqs. 6, 7, 15). We devised a temperature-dependent model of $\gamma$ (Eq. 11) from the thermodynamic identity (Eq. 2). The anharmonic model with only two additional parameters $\left(\theta_{a}\right.$ and $\left.\gamma_{a}\right)$ can reasonably reproduce the properties of $\gamma$ and $C_{V}$ simultaneously in the CTEoS. We examined the applicability limit of the previous linear anharmonic model (Eq. 17), and at high temperatures 


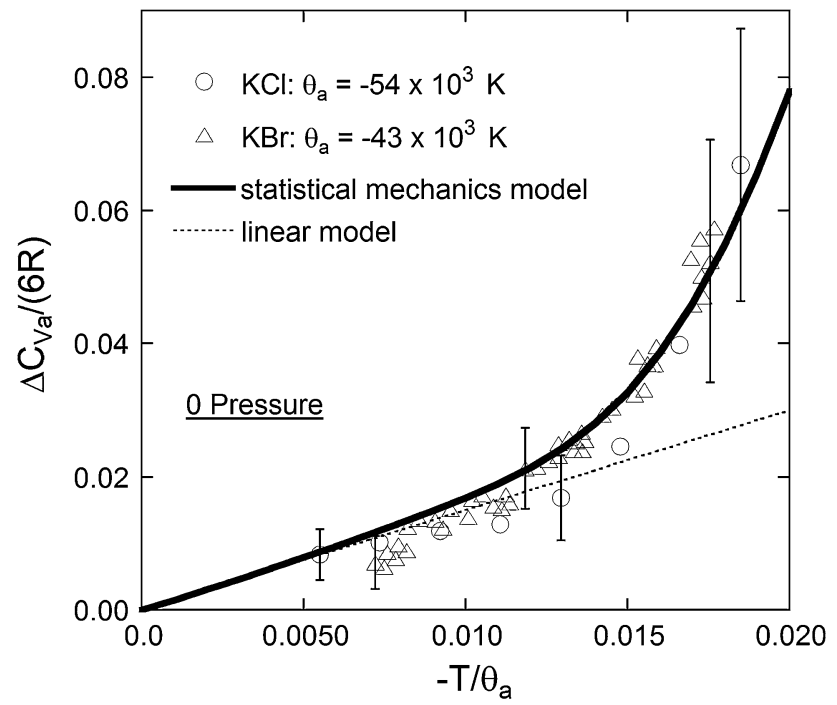

Fig. 15 Intrinsic anharmonic contributions to the specific heat at constant volume for $\mathrm{KCl}$ and $\mathrm{KBr}$. Experimental data and the anharmonic characteristic temperatures $\theta_{a}$ are from Leadbetter et al. (1969). The statistical mechanics model (bold line) is calculated from Eqs. 4 and 21 with the condition $\theta_{a_{6}}=-2.212 \theta_{a}$. The linear model (dotted line) is from Eqs. 17 and 18. Error bars are shown for experimental data

$\left(T / \theta_{a}>10^{-2}\right)$, the linear model optimized with respect to heat capacities $\left(C_{V}\right.$ or $\left.C_{P}\right)$ overestimates $\gamma$.

Acknowledgments We are grateful to Dr. Peter I. Dorogokupets, Dr. Koji Masuda, and Dr. Katsuhiro Tsukimura for their constructive comments and to an anonymous reviewer for comments about the systematic error of the CT-EoS analyses. This paper presents results from a joint research program carried out at the Institute for Study of the Earth's Interior, Okayama University.

Open Access This article is distributed under the terms of the Creative Commons Attribution License which permits any use, distribution, and reproduction in any medium, provided the original author(s) and the source are credited.

\section{Appendix}

Derivation of $\left(\partial C_{V} / \partial \ln V\right)_{T}=C_{V}(\partial \gamma / \partial \ln T)_{V}$ $+\gamma\left(\partial C_{V} / \partial \ln T\right)_{V}$

The relationship between the internal energy $(U)$ and the Helmholtz free energy $(F)$ is

$F=U-T S$,

where $S$ is entropy. Specific heat and pressure can be defined as partial derivatives of the energy:

$C_{V}=(\partial U / \partial T)_{V}$

$P=-(\partial F / \partial V)_{T}$.
Maxwell's thermal pressure relation is

$(\partial S / \partial V)_{T}=(\partial P / \partial T)_{V}=\gamma C_{V} / V$.

Using the above equations, we obtain the volume derivative of the specific heat as

$$
\begin{aligned}
& \left(\frac{\partial C_{V}}{\partial V}\right)_{T}=\left(\frac{\partial U}{\partial T \partial V}\right)=\left(\frac{\partial(F+T S)}{\partial V \partial T}\right) \\
& =-\left(\frac{\partial P}{\partial T}\right)_{V}+\left(\frac{\partial\left(S+T(\partial S / \partial T)_{V}\right)}{\partial V}\right)_{T} \\
& =-\frac{\gamma}{V} C_{V}+\frac{\gamma}{V} C_{V}+\left(\frac{\partial^{2} S}{\partial V \partial \ln T}\right) \\
& \left.\left.=\left(\frac{\partial\left(\gamma C_{V} / V\right)}{\partial \ln T}\right)_{V}\right)_{V}+\gamma\left(\frac{\partial C_{V}}{\partial \ln T}\right)_{V}\right) . \\
& =\frac{1}{V}\left(C_{V}\left(\frac{\partial \gamma}{\partial \ln T}\right)_{V}\right.
\end{aligned}
$$

Thus, we have

$\left(\frac{\partial C_{V}}{\partial \ln V}\right)_{T}=C_{V}\left(\frac{\partial \gamma}{\partial \ln T}\right)_{V}+\gamma\left(\frac{\partial C_{V}}{\partial \ln T}\right)_{V}$.

\section{References}

Anderson OL (1999) The volume dependence of thermal pressure in perovskite and other minerals. Phys Earth Planet Int 112: 267-283

Archer DG (1997) Enthalpy Increment Measurement for $\mathrm{NaCl}(\mathrm{cr})$ and $\mathrm{KBr}$ (cr) from $4.5 \mathrm{~K}$ to $350 \mathrm{~K}$. Thermodynamic properties of the $\mathrm{NaCl}+\mathrm{H}_{2} \mathrm{O}$ system. 3. J Chem Eng Data 42:281-292

Baonza VG, Cáceres M, Núñez J (1995) Universal compressibility behavior of dense phases. Phys Rev B 51:28-37

Birch F (1952) Elasticity and constitution of the Earth's interior. J Geophys Res 57:227-286

Birch F (1986) Equation of state and thermodynamic parameters of $\mathrm{NaCl}$ to $300 \mathrm{kbar}$ in the high-temperature domain. J Geophys Res 91:4949-4954

Boehler R (1981) Adiabats ( $\partial \mathrm{T} / \partial \mathrm{P}) s$ and Grüneisen parameter of $\mathrm{NaCl}$ up to 50 kilobars and $800^{\circ} \mathrm{C}$. J Geophys Res 86:7159-7162

Boehler R, Getting IC, Kennedy GC (1977) Grüneisen parameter of $\mathrm{NaCl}$ at high compressions. J Phys Chem Solids 38:233-236

Brown JM (1999) The $\mathrm{NaCl}$ pressure standard. J Appl Phys 86:5801-5808

Chase MW (1998) NIST-JANAF Thermochemical Tables. Part I, 4th edn. AIP, New York

Cowley ER (1971) Anharmonic contributions to the thermodynamic properties of sodium chloride. J Phys C Solid State Phys 4:988-997

Cuccoli A, Tognetti V, Vaia R (1990) Thermodynamic properties of a quantum chain with nearest-neighbor anharmonic interactions. Phys Rev B 41:R9588-R9591 
Dawson R, Brackett EB, Brackett TE (1963) A high temperature calorimeter; the enthalpies of $\alpha$-aluminum oxide and sodium chloride. J Phys Chem 67:1669-1671

Decker DL (1971) High-pressure equation of state for $\mathrm{NaCl}, \mathrm{KCl}$, and CsCl. J Appl Phys 42:3239-3244

Dorogokupets PI (2002) Critical analysis of equations of state for $\mathrm{NaCl}$. Geochem Inter 40(Supplement 1):S132-S144

Dorogokupets PI, Dewaele A (2007) Equations of state of $\mathrm{MgO}$, $\mathrm{Au}, \mathrm{Pt}, \mathrm{NaCl}-\mathrm{B} 1$, and NaCl-B2: internally consistent hightemperature pressure scales. High Press Res 27:431-446. doi:10.1080/08957950701659700

Enck FD, Dommel JG (1965) Behavior of the thermal expansion of $\mathrm{NaCl}$ at elevated temperatures. J Appl Phys 36:839-844

Holm BJ, Grønvold F (1973) Enthalpies of fusion of the alkali cryolites determined by drop calorimetry. Acta Chem Scand 27:2043-2050

Holzapfel WB (2002) Anharmonicity in the equations of state of $\mathrm{Cu}$, $\mathrm{Ag}$, and $\mathrm{Au}$ and related uncertainties in the realization of a practical pressure scale. J Phys Condens Matter 14:10525-10531

Kirby RK, Hahn TA, Rothrock BD (1972) Thermal expansion. In: Gray DE (ed) American institute of physics handbook, 3rd edn. McGraw-Hill, New York, pp 4-119-4-142

Kittel C (1953) Introduction to solid state physics. Wiley, New York

Leadbetter AJ (1968) Anharmonic effects in the thermodynamic properties of solids II. Analysis of data for lead and aluminium. J Phys C Solid State Phys 1:1489-1504

Leadbetter AJ, Newsham DMT (1969) Anharmonic effects in the thermodynamic properties of solids III. A liquid gallium immersion dilatometer for the range $50-700^{\circ} \mathrm{C}$ : thermal expansivities of $\mathrm{Hg}, \mathrm{Ga}, \mathrm{NaCl}$ and $\mathrm{KCl}$. J Phys C Solid State Phys 2:210-219

Leadbetter AJ, Settatree GR (1969) Anharmonic effects in the thermodynamic properties of solids IV. The heat capacities of $\mathrm{NaCl}$, $\mathrm{KCl}$ and $\mathrm{KBr}$ between 30 and $500^{\circ} \mathrm{C}$. J Phys C Solid State Phys 2:385-392

Leadbetter AJ, Newsham DMT, Settatree GR (1969) Anharmonic effects in the thermodynamic properties of solids V. Analysis of data for $\mathrm{NaCl}, \mathrm{KCl}$ and $\mathrm{KBr}$. J Phys C Solid State Phys 2:393-403

Legge JC, Robinson MC, Shapiro MM (1979) Capacitance determination of the area thermal expansion of dielectric crystals. Rev Sci Instrum 50:832-834

Magnus A (1913) Specific heat measurements of stable solids at high temperatures. Phys Z 14:5-11 (in German)

Matsui M, Higo Y, Okamoto Y, Irifune T, Funakoshi K (2012) Simultaneous sound velocity and density measurements of $\mathrm{NaCl}$ at high temperatures and pressures: application as a primary pressure standard. Am Mineral 97:1670-1675. doi:10.2138/am.2012.4136

Meincke PPM, Graham GM (1965) The thermal expansion of alkali halides. Can J Phys 43:1853-1866

Ming LC, Manghnani MH, Balogh J, Qadri SB, Skelton EF, Jamieson JC (1983) Gold as a reliable internal pressure calibrant at high temperature. J Appl Phys 54:4390-4397

Murnaghan FD (1944) The compressibility of media under extreme pressures. Proc Nat Acad Sci USA 30:244-247

Murphy CA, Jackson JM, Sturhahn W, Chen B (2011) Grüneisen parameter of hcp-Fe to $171 \mathrm{GPa}$. Geophys Res Lett 38:L24306. doi:10.1029/2011GL049531

Nüsslein V, Schröder U (1967) Calculations of dispersion curves and specific heat for $\mathrm{LiF}$ and $\mathrm{NaCl}$ using the breathing shell model. Phys Status Solidi 21:309-314
Oganov AR, Dorogokupets PI (2003) All-electron and pseudpotential study of $\mathrm{MgO}$ : equation of state, anharmonicity, and stability. Phys Rev B 67:224110/1-224110/11. doi: 10.1103/PhysRevB.67.224110

Oganov AR, Dorogokupets PI (2004) Intrinsic anharmonicity in equations of state and thermodynamics of solids. J Phys Condens Matter 16:1351-1360. doi:10.1088/0953-8984/16/8/018

Pathak PD, Vasavada NG (1970) Thermal expansion of $\mathrm{NaCl}, \mathrm{KCl}$ and $\mathrm{CsBr}$ by X-ray diffraction and the law of corresponding states. Acta Crystallogr Sect A 26:655-658

Ponkratz U, Holzapfel WB (2004) Equations of state for wide ranges in pressure and temperature. J Phys Condens Mat 16:S963-S972. doi:10.1088/0953-8984/16/14/005

Powell DGM, Fletcher GC (1965) Thermal expansion and other properties of sodium chloride. Aust J Phys 18:205-217

Rapp JE, Merchant HD (1973) Thermal expansion of alkali halides from 70 to 570 K. J Appl Phys 44:3919-3923

Rössler T, Page JB (1995) Quantum mechanics, quantum-classical correspondence, thermodynamics, and response of a small anharmonic periodic chain. Phys Rev B 51:11382-11392

Roth WA, Bertram W (1929) Measurements of the specific heat of metallurgically important substances in a large temperature interval with help of two new calorimeter types. Z Elektrochem 35:297-308 (in German)

Rubin T, Johnston HL, Altman HW (1961) Thermal expansion of rock salt. J Phys Chem 65:65-68

Schwarz M Jr (1976) Statistical thermodynamics of an anharmonic oscillator. J Stat Phys 15:255-261

Slagle OD, McKinstry HA (1967) Temperature dependence of the elastic constants of the alkali halides. I. $\mathrm{NaCl}, \mathrm{KCl}$, and $\mathrm{KBr}$. J Appl Phys 38:437-446

Spetzler HA, Yoneda A (1993) Performance of the complete traveltime equation of state at simultaneous high pressure and temperature. Pure Appl Geophys 141:379-392

Spetzler H, Sammis CG, O'Connell RJ (1972) Equation of state of $\mathrm{NaCl}$ : ultrasonic measurements to $8 \mathrm{kbar}$ and $800^{\circ} \mathrm{C}$ and static lattice theory. J Phys Chem Solids 33:1727-1750

Spinolo G, Massarotti V, Campari G (1979) A polythermal attachment for X-ray powder diffractometers. J Phys E 12:1059-1062

Stacey FD, Isaak DG (2003) Anharmonicity in mineral physics: a physical interpretation. J Geophys Res 108:2440. doi:10.1029/2 002JB002316

Taravillo M, Baonza VG, Rubio JEF, Núñez J, Cáceres M (2002) The temperature dependence of the equation of state at high pressures revisited: a universal model for solid. J Phys Chem Solids 63:1705-1715

Vinet P, Ferrante J, Rose JH, Smith JR (1987) Compressibility of solids. J Geophys Res 92:9319-9325

White GK (1965) The thermal expansion of alkali halides at low temperatures. Proc R Soc London Ser A 286:204-217

Yamamoto S, Ohno I, Anderson OL (1987) High temperature elasticity of sodium chloride. J Phys Chem Solids 48:143-151

Yates B, Panter CH (1962) Thermal expansion of alkali halides at low temperatures. Proc Phys Soc London 80:373-382

Yoneda A, Osako M, Ito E (2009) Heat capacity measurement under high pressure: a finite element method assessment. Phys Earth Planet Int 174:309-314 